\title{
The health literacy questionnaire among the aged in Changsha, China: confirmatory factor analysis
}

\author{
Yiwei Huang, Tingting Ruan, Qiaoyun Yi* ${ }^{*}$, Tingting Wang and Zhihua Guo
}

\begin{abstract}
Background: Health literacy is defined as the cognitive and social skills that determine the motivation and ability of individuals to gain access to, understand and use information in ways that promote and maintain good health. A Health Literacy Questionnaire (HLQ) is a toolkit with good reliability and validity. Accordingly, this study administered HLQ among older adults in China to examine its factor structure, reliability, homogeneity, and discriminant validity for use in understanding better the health literacy of older adults and determining corresponding measures.

Methods: Psychometric properties were examined based on the data collected via face-to-face interviews $(N=343)$. Tests included the difficulty level, composite scale reliability, confirmatory factor analysis (CFA), and Bayesian structural equation modeling (BSEM).

Results: The easiest scale to obtain a high score was "Social support for health" and the hardest, "Navigating the health care system" and "Appraisal of health information." Two one-factor models fitted well with no correlated residuals allowed. After model modification, the CFA fit statistics of the other seven scales were good. All HLQ scales were found to be homogenous, with a composite reliability ranging from 0.74 to 0.85 . The nine-factor Bayesian structural equation model fitted the data well (Posterior-Predictive-P value $=0.670 ; 95 \%$ Confidence Interval for the difference between the observed and replicated Chi-square values $=-163.320,102.750$ ).

Conclusions: The Chinese version of the HLQ has strong construct and content validity and high composite reliability when applied to older adults in Changsha City, China. Therefore, the nine-scale HLQ can now be administered to Chinese older adults, thereby providing a powerful approach to understanding the multidimensional area of health literacy.
\end{abstract}

Keywords: Health literacy, Questionnaire, HLQ, Bayesian structural equation modeling, BSEM, Psychometric

\section{Background}

The World Health Organization defined health literacy as "the cognitive and social skills which determine the motivation and ability of individuals to gain access to, understand and use information in ways which promote and maintain good health" [1]. The focus on health literacy has intensified over the last two decades, especially in the areas of health systems improvement, public health, and health policy $[2,3]$. In both developing and developed countries, health literacy is a key consideration for promoting health

\footnotetext{
* Correspondence: 2269086514@qq.com

Xiangya School of Nursing, Central South University, Changsha, Hunan, People's Republic of China
}

and improving the quality of health services, owing to the complexity of modern health care and the promotion of health messages [4-8].

Health literacy is important in public health and health care, playing a crucial role in the management of chronic diseases [4]. It can affect health behaviors and health outcomes directly or indirectly and be used as an independent factor in the excessive expenditure of medical expenses $[9,10]$. A study pointed out that when people manage their own health, including the use of various health technologies, having a wide range of health literacy competencies becomes a necessity [11]. Research has shown that most adults will face a situation where their 
health literacy is inadequate in relation to the complexity of the health issues they face [2].

The older adults population is a vulnerable group with regard to health literacy. Research has indicated that functional health literacy is negatively associated with age [12]. According to an adult health literacy survey conducted in the United States, adults in the oldest age group (65 and older) had a lower average health literacy than those in the younger age groups [13]. Similarly, China's first survey on health literacy showed a low health literacy level among older adults, with only $3.81 \%$ of people over 65 years old showing adequate health literacy skills [14]. Limited health literacy causes older adults to have insufficient understanding of chronic diseases, poor self-management of diseases, a low utilization rate of medical resources, and poor compliance with drug treatment. Low health literacy tends to cause stigma among older adults and limit their use of health care services. The implications of older adults being not treated appropriately include their worse general health conditions, their poor health care access, higher admission rates, and higher emergency rates, ultimately leading to an increase in personal and national health expenditure [10, 15-17].

Given this context, carrying out interventions for the older adults population is important to improve their health literacy levels and quality of life. Toward this end, we need to understand the status of their health literacy. However, we need to know that older adults belong to a special category and require appropriate special health literacy assessment tools, which must reflect accurately their situation and be easy to use. Current measures of health literacy focus on a limited range of health-related literacy and numeracy skills [18]. However, it has been demonstrated that health literacy is a multidimensional concept that contains a variety of cognitive, affective, social, and personal skills and attributes $[19,20]$. The study of China's health literacy started relatively late, whereas research on assessment tools lags behind. Currently, the most used health literacy assessment tool is the health literacy questionnaire for Chinese residents. The questionnaire has several items and takes a long time to be completed. The content of the questionnaire includes, among others, infant feeding and HIV prevention, which cannot reflect clearly the characteristics of older adults. There is no mature development in the dimension of critical literacy. Although this tool is commonly used for the evaluation of older adults, some items do not represent their characteristics [21].

Recent developments in the measurement of health literacy have increased its capacity to assess the needs of older adults across a more extensive dimension of health literacy. The Health Literacy Questionnaire (HLQ) was developed in Australia in 2012 using a "validity driven" approach. It was initially tested in diverse individuals in Australian communities, covering nine conceptually distinct areas of health literacy, to assess the needs and challenges of a wide range of people and organizations. It has been shown to have strong construct validity, reliability, and high acceptability to Public Health clients and clinicians [22]. The HLQ has been widely translated and applied in research, evaluation, and monitoring [11]. Daniel et al. used the HLQ to assess the health literacy of older adults with diabetes as a guide to designing health promotion programs [23]. It is envisaged to use the Chinese version of HLQ to measure the strengths and limitations of people in accessing, understanding, and using health information and health services, and determine the health literacy status of Chinese older adults. The data to be derived from this tool can be used to justify, endorse, or exclude treatments, interventions, and policies. Such responsibility requires a measurement tool and its data to be valid for older adults. It is expected that the HLQ will be a suitable tool in China. However, it will be necessary to undertake rigorous studies to confirm its applicability in each setting [24, 25].

\section{Methods \\ Setting}

A cross-sectional survey was carried out from March to May 2018 in the six districts of Changsha City (Yuelu, Tianxin, Kaifu, Furong, Yuhua, and Wangcheng). One or two communities were selected to conduct random sampling surveys of older adults within the scope of each community. The Chinese version of HLQ was used to describe the health literacy profile of and collect general information on older adults in Changsha. Data were collected by researchers through face-to-face interviews. The researchers helped the participants who could not fill out the questionnaire by themselves.

\section{Participants}

Participants included in the study were: (1) aged 60 years and above; (2) residents of Changsha City or having lived in Changsha City for more than six months; (3) voluntary participation. Exclusion criteria were: (1) those with mental illness or confusion; (2) those with a major illness that makes them unable to cooperate; and (3) other investigators who have been involved in a similar investigation.

\section{HLQ}

The questionnaire is divided into nine scales and has 44 items. Each dimension can be used as an assessment tool alone. The sum of the scores is the total score of health literacy. The health literacy is proportional to the score. The nine scales are:

1. Feeling understood and supported by health care providers 
2. Having sufficient information to manage my health

3. Actively managing my health

4. Social support for health

5. Appraisal of health information

6. Ability to actively engage with health care providers

7. Navigating the health care system

8. Ability to find good health information

9. Understand health information well enough to know what to do

\section{Statistical analysis}

Analyses were conducted using IBM SPSS Statistics version 22, Amos version 22.0 and Mplus version 7.4. SPSS was used to provide difficulty estimates within and across the nine scales. Some descriptive statistics were generated for every item, which can determine the range of answers as illustrated by providing difficulty estimates over and across nine levels. As the scales had two types of response options, the difficulty level of each item was calculated through two different methods. The first method, which was applicable to the scales (1-5) with agree/disagree options, was calculated as the ratio of disagree and strongly disagree responses to agree or strongly agree responses. The second method, which was applied to the other scales (6-9), was calculated as the ratio of cannot do, very difficult, or quite difficult responses to quite easy and very easy responses ([11], p., 3 ).

Validity is the degree to which the measured results reflect what is examined. In this study, the Short Form-12 (SF-12) Quality of Life Scale was used as the validity criterion. SPSS was used to calculate the Spearman rank correlation coefficient to observe the relationship between the HLQ questionnaire and calibration.

Reliability is an indicator to measure the stability and accuracy. The most commonly used indicator for evaluating test reliability is Cronbach's $\alpha$. As the $\alpha$ coefficient is often biased in population reliability estimates, it is more reliable to use a confirmatory factor analysis (CFA) to calculate the composite reliability [26]. For this, we used nine one-factor CFA models fitted to the data to confirm the scales. Composite reliability was computed by Amos with robust maximum likelihood estimation [27]. To evaluate the fitness of these models, fit indices "unstandardized and standardized" factor loadings, estimation of variance of measured variables explained by the latent variable $\left(R^{2}\right)$, Root Mean Square Error of Approximation (RMSEA), Tucker-Lewis Index (TLI), and the Comparative Fit Index (CFI) were applied. A value of 0.05 was interpreted as close fit for RMSEA and 0.08 as acceptable fit. For both TLI and CFI, a cut-off value of 0.95 was applied [28-32].
It has been argued that the traditional CFA using maximum likelihood estimation testing applies unnecessarily rigorous models to verify theory hypothesis, which often leads to rejection of the model [33]. For this study, data using the Mplus code was used to investigate whether the discriminant validity fits a specific full nine-factor Bayesian structural equation modeling (BSEM) with no correlated residuals or cross-loadings [34-36]. With the use of small variance priors, BSEM makes it available for models to adapt to flexibility to evaluate minor variations in the rigorous zero-constraints of residual correlations and cross-loadings in a typical multi-factor CFA model [35]. It is thus possible to achieve good model fitting and subsequent unbiased estimation of model parameters.

The BSEM uses a different method from the commonly used ones to assess model fit. After calculating the chisquare likelihood data, a "post-test prediction-P value" (PPP value) was generated to evaluate the model fit. In the well-fitted model, the PPP value was close to 0.5 ; a lower value or a value close to 0.0 indicates poor fit.

\section{Results}

\section{Reliability and homogeneity of the Chinese HLQ scales}

As shown in Table 1, the first five scales, compared with the last four scales, are relatively difficult at the item level. The easiest scale to obtain a high score was "4. Social support for health," with an average item difficulty of 0.15 . The hardest scales to obtain a high score were "7. Navigating the health care system" (0.56) and "8. Ability to find good health information" (0.53). The two easiest items were under " 4 . Social support for health": "4.4 I have at least one person who can come to medical appointments with me" (0.11) and "4.5 I have strong support from family or friends" $(0.11)$. The hardest item was found in scale "7. Navigating the health care system": "7.5 Find out what health care services you are entitled to" (0.70). Scale "5. Appraisal of health information" had the smallest difficulty range (hardest: 0.40, easiest: 0.34, range: 0.06 ), whereas scale " 7 . Navigating the health care system" (hardest: 0.70, easiest: 0.46, range: 0.24) and "9. Understanding health information well enough to know what to do" (hardest: 0.55, easiest: 0.31, range: 0.24 ) had the widest difficulty range (Table 1 ).

In the one-factor models, initially two scales ("2. Having sufficient information to manage own health" and " 8 . Ability to find good health information") were a satisfactorily close fit without a "wiggle room." For the other seven scales, the fit statistics were initially not satisfactory. However, after the model was modified, the model fit for all scales were perfectly fit due to the existence of correlated residuals. These correlation residuals, when contained in the model, occurred at most three times, ranging 
Table 1 Data quality of the translated Health Literacy Questionnaire (HLQ) among the Changsha elder population ( $n=343$ )

\begin{tabular}{|c|c|c|c|c|c|c|c|c|c|}
\hline Subscale/items & $\begin{array}{l}\text { Obs } \\
(n=343)\end{array}$ & $\begin{array}{l}\text { Missing } \\
{[n(\%)]}\end{array}$ & Median & $\begin{array}{l}\text { Mean } \\
(\mathrm{SD})\end{array}$ & $\begin{array}{l}\text { Strongly } \\
\text { disagree (\%) }\end{array}$ & $\begin{array}{l}\text { Disagree } \\
(\%)\end{array}$ & $\begin{array}{l}\text { Agree } \\
(\%)\end{array}$ & $\begin{array}{l}\text { Strongly } \\
\text { agree (\%) }\end{array}$ & $\begin{array}{l}\text { Difficulty level } \\
(\%)(95 \% \mathrm{CL})\end{array}$ \\
\hline \multicolumn{10}{|c|}{ Feeling understood and supported by health care providers } \\
\hline $\begin{array}{l}\text { I have at least one healthcare provider } \\
\text { who knows me well }\end{array}$ & 343 & 0 & 3 & $\begin{array}{l}2.66 \\
(0.70)\end{array}$ & 4.7 & 33.2 & 53.9 & 8.2 & $37.9(36.5-49.1)$ \\
\hline $\begin{array}{l}\text { I have at least one healthcare provider I } \\
\text { can discuss }\end{array}$ & 343 & 0 & 3 & $\begin{array}{l}2.68 \\
(0.67)\end{array}$ & 5.2 & 27.7 & 60.6 & 6.4 & $32.9(30.7-43.0)$ \\
\hline $\begin{array}{l}\text { I have the healthcare providers I need to } \\
\text { help me work }\end{array}$ & 343 & 0 & 3 & $\begin{array}{l}2.67 \\
(0.69)\end{array}$ & 5.8 & 28.0 & 59.5 & 6.7 & $33.8(31.1-43.5)$ \\
\hline \multirow[t]{2}{*}{$\begin{array}{l}\text { I can rely on at least one healthcare } \\
\text { provider }\end{array}$} & 343 & 0 & 3 & $\begin{array}{l}2.61 \\
(0.75)\end{array}$ & 7.6 & 32.4 & 51.6 & 8.5 & $40.0(39.2-53.2)$ \\
\hline & & & & $\begin{array}{l}2.66 \\
(0.70)\end{array}$ & & & & & 36.2 \\
\hline \multicolumn{10}{|c|}{ 2. Having sufficient information to manage my health } \\
\hline $\begin{array}{l}\text { I feel I have good information about } \\
\text { health }\end{array}$ & 343 & 0 & 3 & $\begin{array}{l}2.80 \\
(0.62)\end{array}$ & 2.9 & 22.4 & 66.8 & 7.9 & $25.3(22.9-34.3)$ \\
\hline $\begin{array}{l}\text { I have enough information to help me } \\
\text { deal with my }\end{array}$ & 343 & 0 & 3 & $\begin{array}{l}2.60 \\
(0.62)\end{array}$ & 1.7 & 42 & 50.7 & 5.5 & $43.7(40.6-52.3))$ \\
\hline $\begin{array}{l}\text { I am sure I have all the information I } \\
\text { need to manage }\end{array}$ & 343 & 0 & 3 & $\begin{array}{l}2.59 \\
(0.68)\end{array}$ & 3.8 & 40.5 & 48.4 & 7.3 & $44.3(39.7-52.0)$ \\
\hline \multirow[t]{2}{*}{$\begin{array}{l}\text { I have all the information I need to look } \\
\text { after my health }\end{array}$} & 343 & 0 & 3 & $\begin{array}{l}2.58 \\
(0.68)\end{array}$ & 4.1 & 41.1 & 47.8 & 7.0 & $45.2(39.8-52.5)$ \\
\hline & & & & $\begin{array}{l}2.64 \\
(0.65)\end{array}$ & & & & & 39.6 \\
\hline \multicolumn{10}{|l|}{ 3. Actively managing my health } \\
\hline $\begin{array}{l}\text { I spend quite a lot of time actively } \\
\text { managing my health }\end{array}$ & 343 & 0 & 3 & $\begin{array}{l}2.77 \\
(0.66)\end{array}$ & 1.5 & 31.2 & 56.0 & 11.4 & $32.7(30.2-41.0)$ \\
\hline $\begin{array}{l}\text { I make plans for what I need to do to be } \\
\text { healthy. }\end{array}$ & 343 & 0 & 3 & $\begin{array}{l}2.60 \\
(0.78)\end{array}$ & 9.9 & 28.9 & 52.8 & 8.5 & $38.8(33.5-48.0)$ \\
\hline $\begin{array}{l}\text { Despite other things in my life, I make } \\
\text { time to be healthy }\end{array}$ & 343 & 0 & 3 & $\begin{array}{l}2.83 \\
(0.64)\end{array}$ & 2.3 & 23.6 & 63.0 & 11.1 & $25.9(23.4-34.3)$ \\
\hline $\begin{array}{l}\text { I set my own goals about health and } \\
\text { fitness }\end{array}$ & 343 & 0 & 3 & $\begin{array}{l}2.62 \\
(0.74)\end{array}$ & 8.2 & 29.2 & 55.4 & 7.3 & $37.4(31.1-44.6)$ \\
\hline \multirow[t]{2}{*}{$\begin{array}{l}\text { There are things that I do regularly to } \\
\text { make myself more healthy }\end{array}$} & 343 & 0 & 3 & $\begin{array}{l}2.87 \\
(0.63)\end{array}$ & 2.3 & 19.5 & 66.5 & 11.7 & $21.8(18.0-28.4)$ \\
\hline & & & & $\begin{array}{l}2.74 \\
(0.69)\end{array}$ & & & & & 31.3 \\
\hline \multicolumn{10}{|l|}{ 4. Social support for health } \\
\hline $\begin{array}{l}\text { I can get access to several people who } \\
\text { understand and ... }\end{array}$ & 343 & 0 & 3 & $\begin{array}{l}3.03 \\
(0.61)\end{array}$ & 0.3 & 16.6 & 63.3 & 19.8 & $16.9(15.8-23.2)$ \\
\hline $\begin{array}{l}\text { When I feel ill, the people around me } \\
\text { really understand }\end{array}$ & 343 & 0 & 3 & $\begin{array}{l}2.99 \\
(0.58)\end{array}$ & 0.9 & 14.6 & 69.1 & 15.5 & $15.5(12.8-21.6)$ \\
\hline $\begin{array}{l}\text { If I need help, I have plenty of people I } \\
\text { can rely on. }\end{array}$ & 343 & 0 & 3 & $\begin{array}{l}2.94 \\
(0.73)\end{array}$ & 3.2 & 20.1 & 56.6 & 20.1 & $23.3(20.8-32.2)$ \\
\hline $\begin{array}{l}\text { I have at least one person who can } \\
\text { come to medical }\end{array}$ & 343 & 0 & 3 & $\begin{array}{l}3.15 \\
(0.63)\end{array}$ & 1.5 & 9.3 & 62.4 & 26.8 & $10.8(9.7-18.1)$ \\
\hline \multirow[t]{2}{*}{$\begin{array}{l}\text { I have strong support from family or } \\
\text { friends. }\end{array}$} & 343 & 0 & 3 & $\begin{array}{l}3.13 \\
(0.62)\end{array}$ & 1.5 & 9.0 & 65.0 & 24.5 & $10.5(8.8-16.9)$ \\
\hline & & & & $\begin{array}{l}3.05 \\
(0.63)\end{array}$ & & & & & 15.4 \\
\hline \multicolumn{10}{|l|}{ 5. Appraisal of health information } \\
\hline $\begin{array}{l}\text { I compare health information from } \\
\text { different sources }\end{array}$ & 343 & 0 & 3 & $\begin{array}{l}2.73 \\
(0.67)\end{array}$ & 2.9 & 30.6 & 56.6 & 9.9 & $33.5(28.4-40.8)$ \\
\hline When I see new information about & 343 & 0 & 3 & 2.64 & 5.5 & 31.8 & 55.7 & 7.0 & $37.3(32.7-45.7)$ \\
\hline
\end{tabular}


Table 1 Data quality of the translated Health Literacy Questionnaire (HLQ) among the Changsha elder population ( $\mathrm{n}=343$ ) (Continued)

\begin{tabular}{|c|c|c|c|c|c|c|c|c|c|c|}
\hline Subscale/items & $\begin{array}{l}\text { Obs } \\
(n=343)\end{array}$ & $\begin{array}{l}\text { Missing } \\
{[n(\%)]}\end{array}$ & Median & $\begin{array}{l}\text { Mean } \\
(\mathrm{SD})\end{array}$ & $\begin{array}{l}\text { Strongly } \\
\text { disagree (\%) }\end{array}$ & $\begin{array}{l}\text { Disagree } \\
(\%)\end{array}$ & $\begin{array}{l}\text { Agree } \\
(\%)\end{array}$ & $\begin{array}{l}\text { Strongly } \\
\text { agree (\%) }\end{array}$ & \multicolumn{2}{|c|}{$\begin{array}{l}\text { Difficulty level } \\
(\%)(95 \% \mathrm{CL})\end{array}$} \\
\hline \multicolumn{4}{|l|}{ health, I check up } & \multicolumn{7}{|l|}{$(0.69)$} \\
\hline $\begin{array}{l}\text { I always compare health information } \\
\text { from different sources }\end{array}$ & 343 & 0 & 3 & $\begin{array}{l}2.62 \\
(0.69)\end{array}$ & 4.7 & 35.6 & 52.8 & 7.0 & \multicolumn{2}{|c|}{$40.3(34.9-48.0)$} \\
\hline $\begin{array}{l}\text { I know how to find out if the health } \\
\text { information I receive is }\end{array}$ & 343 & 0 & 3 & $\begin{array}{l}2.60 \\
(0.69)\end{array}$ & 6.4 & 32.4 & 55.7 & 5.5 & \multicolumn{2}{|c|}{$38.8(34.0-47.3)$} \\
\hline \multirow[t]{2}{*}{$\begin{array}{l}\text { I ask healthcare providers about the } \\
\text { quality of the }\end{array}$} & 343 & 0 & 3 & $\begin{array}{l}2.70 \\
(0.71)\end{array}$ & 5.0 & 29.7 & 55.4 & 9.9 & \multicolumn{2}{|c|}{$34.7(31.6-44.1)$} \\
\hline & & & & $\begin{array}{l}2.66 \\
(0.69)\end{array}$ & & & & & \multicolumn{2}{|l|}{36.9} \\
\hline \multicolumn{11}{|c|}{ 6. Ability to actively engage with healthcare providers } \\
\hline $\begin{array}{l}\text { Make sure that healthcare providers } \\
\text { understand your ... }\end{array}$ & 343 & 0 & 4 & $\begin{array}{l}3.39 \\
(0.88)\end{array}$ & 2.6 & 12.0 & 35.3 & 43.7 & 6.4 & $\begin{array}{l}49.9 \\
(44.6- \\
62.7)\end{array}$ \\
\hline $\begin{array}{l}\text { Feel able to discuss your health } \\
\text { concerns with a ... }\end{array}$ & 343 & 0 & 4 & $\begin{array}{l}3.39 \\
(0.92)\end{array}$ & 3.2 & 13.1 & 32.4 & 43.7 & 7.6 & $\begin{array}{l}48.7 \\
(43.1- \\
61.7)\end{array}$ \\
\hline $\begin{array}{l}\text { Have good discussions about your } \\
\text { health with doctors }\end{array}$ & 343 & 0 & 3 & $\begin{array}{l}3.27 \\
(0.90)\end{array}$ & 2.6 & 15.7 & 40.5 & 34.4 & 6.7 & $\begin{array}{l}58.8 \\
(53.2- \\
72.2)\end{array}$ \\
\hline $\begin{array}{l}\text { Discuss things with healthcare providers } \\
\text { until you understand ... }\end{array}$ & 343 & 0 & 3 & $\begin{array}{l}3.31 \\
(0.89)\end{array}$ & 4.1 & 11.1 & 40.2 & 39.1 & 5.5 & $\begin{array}{l}55.4 \\
(49.8- \\
68.3)\end{array}$ \\
\hline \multirow[t]{2}{*}{$\begin{array}{l}\text { Ask healthcare providers questions to } \\
\text { get the }\end{array}$} & 343 & 0 & 4 & $\begin{array}{l}3.47 \\
(0.84)\end{array}$ & 1.2 & 11.1 & 35.6 & 44.0 & 8.2 & $\begin{array}{l}47.9 \\
(43.3- \\
60.0)\end{array}$ \\
\hline & & & & $\begin{array}{l}3.37 \\
(0.89)\end{array}$ & & & & & & 52.1 \\
\hline \multicolumn{11}{|l|}{ 7. Navigating the healthcare system } \\
\hline Find the right healthcare & 343 & 0 & 4 & $\begin{array}{l}3.34 \\
(0.93)\end{array}$ & 2.6 & 19.0 & 25.4 & 47.8 & 5.2 & $\begin{array}{l}47.0 \\
(42.0- \\
61.3)\end{array}$ \\
\hline $\begin{array}{l}\text { Get to see the healthcare providers I } \\
\text { need to }\end{array}$ & 343 & 0 & 3 & $\begin{array}{l}2.95 \\
(1.12)\end{array}$ & 11.4 & 23.6 & 31.2 & 26.5 & 7.3 & $\begin{array}{l}66.2 \\
(57.5- \\
80.7)\end{array}$ \\
\hline $\begin{array}{l}\text { decide which healthcare provider you } \\
\text { need to see }\end{array}$ & 343 & 0 & 3 & $\begin{array}{l}3.32 \\
(0.87)\end{array}$ & 1.7 & 15.5 & 38.5 & 37.9 & 6.4 & $\begin{array}{l}55.7 \\
(49.1- \\
67.0)\end{array}$ \\
\hline $\begin{array}{l}\text { Make sure you find the right place to get } \\
\text { the health ... }\end{array}$ & 343 & 0 & 4 & $\begin{array}{l}3.47 \\
(0.84)\end{array}$ & 0.9 & 12.5 & 32.9 & 45.8 & 7.9 & $\begin{array}{l}46.3 \\
(40.3- \\
57.5)\end{array}$ \\
\hline $\begin{array}{l}\text { Find out what healthcare services you } \\
\text { are entitled to }\end{array}$ & 343 & 0 & 3 & $\begin{array}{l}2.88 \\
(1.10)\end{array}$ & 12.5 & 20.7 & 36.7 & 25.9 & 4.1 & $\begin{array}{l}69.9 \\
(59.9- \\
82.5)\end{array}$ \\
\hline \multirow[t]{2}{*}{ Work out what is the best care for you } & 343 & 0 & 3 & $\begin{array}{l}3.39 \\
(0.92)\end{array}$ & 2.3 & 14.9 & 33.2 & 40.8 & 8.7 & $\begin{array}{l}50.4 \\
(46.1- \\
64.0)\end{array}$ \\
\hline & & & & $\begin{array}{l}3.23 \\
(0.96)\end{array}$ & & & & & & 55.9 \\
\hline \multicolumn{11}{|l|}{ 8. Ability to find good health information } \\
\hline Find information about health problems & 343 & 0 & 3 & $\begin{array}{l}3.36 \\
(0.91)\end{array}$ & 3.8 & 11.4 & 37.6 & 39.9 & 7.3 & $\begin{array}{l}52.8 \\
(45.7- \\
64.7)\end{array}$ \\
\hline $\begin{array}{l}\text { Find health information from several } \\
\text { different places }\end{array}$ & 343 & 0 & 3 & $\begin{array}{l}3.30 \\
(0.96)\end{array}$ & 2.6 & 18.1 & 35.3 & 35.0 & 9.0 & $\begin{array}{l}56.0 \\
(49.0-\end{array}$ \\
\hline
\end{tabular}


Table 1 Data quality of the translated Health Literacy Questionnaire (HLQ) among the Changsha elder population $(n=343)$ (Continued)

\begin{tabular}{|c|c|c|c|c|c|c|c|c|c|c|}
\hline Subscale/items & $\begin{array}{l}\text { Obs } \\
(n=343)\end{array}$ & $\begin{array}{l}\text { Missing } \\
{[n(\%)]}\end{array}$ & Median & $\begin{array}{l}\text { Mean } \\
\text { (SD) }\end{array}$ & $\begin{array}{l}\text { Strongly } \\
\text { disagree (\%) }\end{array}$ & $\begin{array}{l}\text { Disagree } \\
(\%)\end{array}$ & $\begin{array}{l}\text { Agree } \\
(\%)\end{array}$ & $\begin{array}{l}\text { Strongly } \\
\text { agree (\%) }\end{array}$ & \multicolumn{2}{|c|}{$\begin{array}{l}\text { Difficulty level } \\
(\%)(95 \% \mathrm{CL})\end{array}$} \\
\hline & & & & & & & & & & 68.5) \\
\hline $\begin{array}{l}\text { Get information about health so you are } \\
\text { up to date... }\end{array}$ & 343 & 0 & 3 & $\begin{array}{l}3.08 \\
(0.98)\end{array}$ & 5.0 & 23.3 & 37.3 & 28.0 & 6.4 & $\begin{array}{l}65.6 \\
(57.0- \\
78.2)\end{array}$ \\
\hline $\begin{array}{l}\text { Get health information in words you } \\
\text { understand }\end{array}$ & 343 & 0 & 4 & $\begin{array}{l}3.50 \\
(0.82)\end{array}$ & 0.9 & 11.7 & 31.5 & 49.0 & 7.0 & $\begin{array}{l}44.1 \\
(38.3- \\
55.7)\end{array}$ \\
\hline \multirow[t]{2}{*}{ Get health information by yourself } & 343 & 0 & 4 & $\begin{array}{l}3.47 \\
(0.76)\end{array}$ & 0.3 & 9.9 & 37.6 & 46.6 & 5.5 & $\begin{array}{l}47.8 \\
(41.9- \\
57.7)\end{array}$ \\
\hline & & & & $\begin{array}{l}3.34 \\
(0.89)\end{array}$ & & & & & & 53.3 \\
\hline \multicolumn{11}{|c|}{ 9. Understanding health information well enough to know what to do } \\
\hline $\begin{array}{l}\text { Confidently fill medical forms in the } \\
\text { correct way }\end{array}$ & 343 & 0 & 4 & $\begin{array}{l}3.53 \\
(0.91)\end{array}$ & 3.2 & 7.6 & 34.1 & 43.4 & 11.7 & $\begin{array}{l}44.9 \\
(38.8- \\
55.9)\end{array}$ \\
\hline $\begin{array}{l}\text { Accurately follow the instructions from } \\
\text { healthcare providers }\end{array}$ & 343 & 0 & 4 & $\begin{array}{l}3.78 \\
(0.83)\end{array}$ & 0.6 & 6.7 & 24.2 & 51.3 & 17.2 & $\begin{array}{l}31.5 \\
(26.2- \\
41.2)\end{array}$ \\
\hline $\begin{array}{l}\text { Read and understand written health } \\
\text { information }\end{array}$ & 343 & 0 & 3 & $\begin{array}{l}3.36 \\
(0.93)\end{array}$ & 3.2 & 14.3 & 33.8 & 40.8 & 7.9 & $\begin{array}{l}51.3 \\
(43.6- \\
62.7)\end{array}$ \\
\hline $\begin{array}{l}\text { Read and understand all the information } \\
\text { on medication labels }\end{array}$ & 343 & 0 & 3 & $\begin{array}{l}3.30 \\
(0.87)\end{array}$ & 3.2 & 13.1 & 38.5 & 40.5 & 4.7 & $\begin{array}{l}54.8 \\
(48.2- \\
67.1)\end{array}$ \\
\hline \multirow[t]{2}{*}{$\begin{array}{l}\text { Understand what healthcare providers } \\
\text { are asking you to do }\end{array}$} & 343 & 0 & 4 & $\begin{array}{l}3.76 \\
(0.79)\end{array}$ & 0.6 & 5.8 & 24.8 & 54.2 & 14.6 & $\begin{array}{l}31.2 \\
(28.4- \\
42.5)\end{array}$ \\
\hline & & & & $\begin{array}{l}3.55 \\
(0.87)\end{array}$ & & & & & & 42.7 \\
\hline
\end{tabular}

from 0.17 (scale 9) to 0.45 (scale 7). Regarding factor loadings, almost all scales had high loadings for each item at 0.45 or higher, except for one. The median loading was 0.675 , which showed that almost all items were closely related to the structure of the hypothesis [37]. The one exception was in scale "7. Find out what health care services you are entitled to" (0.20).

Table 2 shows that the internal consistency of all HLQ scales was high. In addition to scale "9. Understanding health information well enough to know what to do" (composite reliability $=0.74, \alpha=0.77$ ), composite reliability and Cronbach's $\alpha \geq 0.75$ were observed in all scales. The median composite reliability was $0.78(\alpha=0.78)$. In detail, these were: scale " 2 . Having sufficient information to manage own health" (composite reliability $=0.78, \alpha=0.78$ ); scale " 3 . Actively managing own health" (composite reliability $=0.77$, $\alpha=0.78$ ), scale "4. Social support for health" (composite reliability $=0.81, \alpha=0.81$ ), scale " 5 . Appraisal of health information" (composite reliability $=0.79, \alpha=0.80$ ); scale "6. Ability to actively engage with health care providers" (composite reliability $=0.84, \alpha=0.84$ ), scale " 7 . Navigating the health care system" (composite reliability $=0.75, \alpha=0.75$ ); scale " 8 . Ability to find good health information" (composite reliability $=0.78, \alpha=0.78$ ), and scale " 1 . Feeling understood and supported by health care providers" (composite reliability $=0.85, \alpha=0.86$ ), which was the highest (Table 2 ).

\section{Criterion validity of the Chinese HLQ scales}

The correlation coefficient between the total score of the HLQ questionnaire and the validity criterion was 0.129 . Table 3 shows that the correlation coefficient between the scores of nine scales and the calibration is between 0.100 and 0.191 . And the correlations of the HLQ with the two composite scores that make up the SF-12 were 0.113 and 0.110 . The difference was statistically significant (Table 3).

\section{Factor correlations of the nine factors}

As shown in Table 4, inter-factor correlations between the nine HLQ factors ranged from 0.18 to 0.98 , suggesting satisfactory discrimination, with the possible exception of scales $6,7,8$, and 9, where the inter-factor 
Table 2 Psychometric properties of Chinese-version HLQ items and scales

\begin{tabular}{|c|c|c|c|c|}
\hline \multirow{2}{*}{$\begin{array}{l}\text { Item } \\
\text { 1. Feeling understood and supported by health care providers }\end{array}$} & \multicolumn{2}{|c|}{ Factor loading (95\% CL) } & \multirow[t]{2}{*}{$\mathrm{R}^{2}$} & \multirow{2}{*}{$\begin{array}{l}\text { Composite reliability }(95 \% \mathrm{Cl}) \\
\text { Cronbach's a (italics) } \\
\mathrm{CR}=0.85 \mathrm{a}=0.86\end{array}$} \\
\hline & & & & \\
\hline I have at least one healthcare provider who knows me well & 0.68 & $0.55-0.77$ & 0.46 & \\
\hline I have at least one healthcare provider I can discuss ... & 0.70 & $0.60-0.79$ & 0.49 & \\
\hline I have the healthcare providers I need to help me work & 0.79 & $0.72-0.86$ & 0.63 & \\
\hline I can rely on at least one healthcare provider & 0.87 & $0.81-0.93$ & 0.76 & \\
\hline \multicolumn{5}{|l|}{$\begin{array}{l}\text { Fit with } 1 \text { correlated residual }-\mathrm{x} 2(\mathrm{ML})=0.528, \mathrm{p}=0.468 \text {, } \\
C F \mathrm{I}=1.000, T L \mathrm{I}=1.005, \mathrm{RMSEA}=0.000\end{array}$} \\
\hline 2. Having sufficient information to manage my health & & & & $C R=0.78 a=0.78$ \\
\hline I feel I have good information about health & 0.51 & $0.39-0.61$ & 0.26 & \\
\hline I have enough information to help me deal with my ... & 0.76 & $0.68-0.84$ & 0.58 & \\
\hline I am sure I have all the information I need to manage & 0.72 & $0.60-0.83$ & 0.52 & \\
\hline I have all the information I need to look after my health & 0.75 & $0.65-0.83$ & 0.56 & \\
\hline \multicolumn{5}{|l|}{$\begin{array}{l}\text { Model fit—X2 (ML) }=5.427, p=0.066, C F I=0.991, T L I=0.973 \\
\text { RMSEA }=0.071\end{array}$} \\
\hline 3. Actively managing my health & & & & $C R=0.77 a=0.78$ \\
\hline I spend quite a lot of time actively managing my health & 0.75 & $0.65-0.83$ & 0.56 & \\
\hline I make plans for what I need to do to be healthy. & 0.47 & $0.33-0.59$ & 0.23 & \\
\hline Despite other things in my life, I make time to be healthy & 0.81 & $0.72-0.88$ & 0.65 & \\
\hline I set my own goals about health and fitness & 0.48 & $0.33-0.61$ & 0.23 & \\
\hline There are things that I do regularly to make myself more healthy & 0.65 & $0.52-0.75$ & 0.43 & \\
\hline \multicolumn{5}{|l|}{$\begin{array}{l}\text { Fit with } 1 \text { correlated residual }-\times 2(\mathrm{ML})=9.566, \mathrm{p}=0.048 \text {, } \\
\mathrm{CFI}=0.989, \mathrm{TLI}=0.972, \mathrm{RMSEA}=0.064\end{array}$} \\
\hline 4. Social support for health & & & & $C R=0.81 a=0.81$ \\
\hline I can get access to several people who understand and & 0.53 & $0.41-0.64$ & 0.28 & \\
\hline When I feel ill, the people around me really understand & 0.62 & $0.52-0.72$ & 0.39 & \\
\hline If I need help, I have plenty of people I can rely on. & 0.66 & $0.59-0.76$ & 0.44 & \\
\hline I have at least one person who can come to medical ... & 0.76 & $0.68-0.83$ & 0.58 & \\
\hline I have strong support from family or friends. & 0.78 & $0.71-0.85$ & 0.61 & \\
\hline \multicolumn{5}{|l|}{$\begin{array}{l}\text { Fit with one correlated residuals }-x 2(\mathrm{ML})=5.143, \mathrm{p}=0.2734 \text {, } \\
\mathrm{CFI}=0.998, \mathrm{TLI}=0.994, \mathrm{RMSEA}=0.029\end{array}$} \\
\hline 5. Appraisal of health information & & & & $C R=0.79 a=0.80$ \\
\hline I compare health information from different sources & 0.63 & $0.52-0.71$ & 0.39 & \\
\hline When I see new information about health, I check up ... & 0.68 & $0.56-0.77$ & 0.47 & \\
\hline I always compare health information from different sources ... & 0.73 & $0.65-0.81$ & 0.54 & \\
\hline I know how to find out if the health information I receive is & 0.71 & $0.62-0.79$ & 0.50 & \\
\hline I ask healthcare providers about the quality of the ... & 0.52 & $0.40-0.62$ & 0.27 & \\
\hline \multicolumn{5}{|l|}{$\begin{array}{l}\text { Fit with one correlated residual }-\times 2(\mathrm{ML})=6.630, p=0.157 \text {, } \\
C F I=0.986, \mathrm{TLI}=0.994, \mathrm{RMSEA}=0.044\end{array}$} \\
\hline 6. Ability to actively engage with healthcare providers & & & & $C R=0.84 a=0.84$ \\
\hline Make sure that healthcare providers understand your ... & 0.78 & $0.71-0.84$ & 0.62 & \\
\hline Feel able to discuss your health concerns with a ... & 0.83 & $0.76-0.89$ & 0.69 & \\
\hline Have good discussions about your health with doctors & 0.78 & $0.72-0.83$ & 0.61 & \\
\hline Discuss things with healthcare providers until you understand. & 0.63 & $0.52-0.72$ & 0.40 & \\
\hline Ask healthcare providers questions to get the ... & 0.56 & $0.44-0.66$ & 0.32 & \\
\hline \multicolumn{5}{|l|}{$\begin{array}{l}\text { Fit with one correlated residual }-x 2(\mathrm{ML})=8.928, \mathrm{p}=0.063 \text {, } \\
\mathrm{CFI}=0.981, \mathrm{TLI}=0.998, \mathrm{RMSEA}=0.060\end{array}$} \\
\hline 7. Navigating the healthcare system & & & & $C R=0.75 a=0.75$ \\
\hline Find the right healthcare & 0.59 & $0.50-0.67$ & 0.35 & \\
\hline Get to see the healthcare providers I need to & 0.52 & $0.41-0.63$ & 0.28 & \\
\hline Decide which healthcare provider you need to see & 0.78 & $0.69-0.85$ & 0.61 & \\
\hline
\end{tabular}


Table 2 Psychometric properties of Chinese-version HLQ items and scales (Continued)

\begin{tabular}{|c|c|c|c|c|}
\hline \multirow{2}{*}{$\begin{array}{l}\text { Item } \\
\text { Make sure you find the right place to get the health ... }\end{array}$} & \multicolumn{2}{|c|}{ Factor loading $(95 \% \mathrm{CL})$} & \multirow{2}{*}{$\begin{array}{l}R^{2} \\
0.51\end{array}$} & \multirow{2}{*}{$\begin{array}{l}\text { Composite reliability }(95 \% \mathrm{Cl}) \\
\text { Cronbach's a (italics) }\end{array}$} \\
\hline & 0.71 & $0.59-0.81$ & & \\
\hline Find out what healthcare services you are entitled to & 0.20 & $0.06-0.35$ & 0.04 & \\
\hline Work out what is the best care for you & 0.59 & $0.48-0.69$ & 0.35 & \\
\hline \multicolumn{5}{|l|}{$\begin{array}{l}\text { Fit with three correlated residuals }-x 2(\mathrm{ML})=9.525, \mathrm{p}=0.146 \text {, } \\
\mathrm{CFI}=0.993, \mathrm{TLI}=0.983, \mathrm{RMSEA}=0.041\end{array}$} \\
\hline 8. Ability to find good health information & & & & $C R=0.78 a=0.78$ \\
\hline Find information about health problems & 0.67 & $0.55-0.75$ & 0.44 & \\
\hline Find health information from several different places & 0.75 & $0.65-0.83$ & 0.56 & \\
\hline Get information about health so you are up to date ... & 0.64 & $0.54-0.71$ & 0.40 & \\
\hline Get health information in words you understand & 0.59 & $0.48-0.69$ & 0.34 & \\
\hline Get health information by yourself & 0.59 & $0.48-0.68$ & 0.34 & \\
\hline \multicolumn{5}{|l|}{$\begin{array}{l}\text { Model fit- } \times 2(\mathrm{ML})=7.502, p=0.186, C F \mid=0.994, T L I=0.988 \\
\text { RMSEA }=0.038\end{array}$} \\
\hline 9. Understanding health information well enough to know what to do & & & & $C R=0.74 a=0.77$ \\
\hline Confidently fill medical forms in the correct way & 0.62 & $0.52-0.71$ & 0.39 & \\
\hline Accurately follow the instructions from healthcare providers & 0.46 & $0.35-0.58$ & 0.21 & \\
\hline Read and understand written health information & 0.76 & $0.64-0.856$ & 0.57 & \\
\hline Read and understand all the information on medication labels & 0.69 & $0.59-0.78$ & 0.48 & \\
\hline Understand what healthcare providers are asking you to do & 0.44 & $0.31-0.55$ & 0.19 & \\
\hline
\end{tabular}

Fit with two correlated residual $-\chi 2(\mathrm{ML})=5.978, p=0.113, \mathrm{CFI}=0.977, \mathrm{TLI}=0.998, \mathrm{RMSEA}=0.054$

correlations were $>0.80$. For scales $2 / 5=0.90,6 / 7=0.98$, $6 / 8=0.82,7 / 8=0.95,7 / 9=0.84$, and $8 / 9=0.89$. This indicated that scales $6,7,8$, and 9 were lacking in discriminant validity (Table 4).

\section{BSEM}

To validate the previously established HLQ factor structure, a Bayesian analysis was used to fit the nine-factor model to the data. For this Bayesian analysis, the variance of the priors for the cross-loadings was set at 0.02 after several attempts, giving a $95 \%$ probability that the cross-loadings would be in the range \pm 0.28 . Similarly, there was also a $95 \%$ probability that the variance for the residual correlations was set at 0.02 ([35], p., 317). As a result, the model resulted in a satisfactory fit $(\mathrm{PPP}=$
0.670; 95\% CI for the difference between the observed and replicated Chi-square values $=-163.320,102.750$ ).

Table 5 shows the pattern of the statistically significant "target and non-target" factor loadings from the Bayesian analysis. The results showed that two scales ("5. Appraisal of health information" and "9. Understanding health information well enough to know what to do") were unifactorial, with two or one statistically significant non-target loadings. Varying items of the other seven scales had statistically significant non-target loadings, indicating some multi-factorial. However, four of these scales (scale 1, 6, 7, and 8) had a pattern of strong target loadings and four statistically significant but small non-target loadings. All non-target loadings were smaller than 0.29 , except for three loadings. Scale "4. Social support for health" had the strongest non-

Table 3 Correlation analysis between the validity criterion and the score of the HLQ

\begin{tabular}{lll}
\hline Scales & correlation coefficient & $P$ \\
\hline Total score of the HLQ & 0.129 & 0.011 \\
1. Feeling understood and supported by healthcare providers & 0.191 & 0.000 \\
2. Having sufficient information to manage my health & 0.159 & 0.002 \\
3. Actively managing my health & 0.113 & 0.026 \\
4. Social support for health & 0.122 & 0.016 \\
5. Appraisal of health information & 0.112 & 0.027 \\
6. Ability to actively engage with healthcare providers & 0.164 & 0.001 \\
7. Navigating the healthcare system & 0.100 & 0.048 \\
8. Ability to find good health information & 0.112 & 0.028 \\
9. Understand health information well enough to know what to do & 0.130 & 0.010 \\
\hline
\end{tabular}


Table 4 Factor correlations of the nine factors of the Chinese

\begin{tabular}{|c|c|c|c|c|c|c|c|c|}
\hline \multirow[t]{2}{*}{ Scale } & \multicolumn{5}{|c|}{ Part I scales } & \multicolumn{3}{|c|}{ Part II scales } \\
\hline & 1 & 2 & 3 & 4 & 5 & 6 & 7 & 8 \\
\hline 2 & .582 & & & & & & & \\
\hline 3 & .389 & .793 & & & & & & \\
\hline 4 & .327 & .228 & .318 & & & & & \\
\hline 5 & .606 & $.899^{\mathrm{a}}$ & .677 & .345 & & & & \\
\hline 6 & .379 & .287 & .221 & .420 & .373 & & & \\
\hline 7 & .362 & .460 & .353 & .335 & .519 & $.978^{\mathrm{a}}$ & & \\
\hline 8 & .352 & .600 & .507 & .363 & 691 & $.817^{a}$ & $.948^{\mathrm{a}}$ & \\
\hline 9 & .176 & .374 & .290 & .493 & .443 & .795 & .835 & $.885^{\mathrm{a}}$ \\
\hline
\end{tabular}

avalues in table indicates high factor correlations

target loadings. The item P2Q13 (i.e., "Make sure you find the right place to get the health care you need") seemed to have the most complex factor structure; it was significantly related to four factors beyond its hypothetical target construct (Table 5).

\section{Discussion}

In this study, we measured the psychological measurement properties of the Chinese version of HLQ using older adults in Changsha as participants. The translated HLQ has a strong strength psychometric structure and original reliability. Since HLQ was not originally designed for older adults who have special characteristics, we need to understand the difficulty of the content items for older adults. In terms of the difficulty level, scale "7. Navigating the health care system" (0.56) was the most difficult among the nine scales. This is consistent with a Danish HLQ study, which found scale $7(0.36)$ to have the highest difficulty level. However, the numerical value is smaller than this study's 0.56 [11]. The difficulty level of scale "8. Ability to find good health information" was also large. The fifth item in scale "7. Find out what health care services you are entitled to" had a difficulty degree of 0.70 . In today's information society, the access to health care services and health information is limited for older adults. Low rates of online health information seeking are reported among them. Their source of information is relatively single. Studies have shown that the trend of using the internet has increased significantly, but for older adults, TV remains their most common source of health information. Overreliance on the traditional medium of television is not conducive to multi-contact and multi-source verification of information [38, 39]. A recent study that used two of the scales from the Danish HLQ showed that older adults may be more deficient in health literacy skills [40]. In short, some of the items in the
Chinese version of the HLQ are a little difficult for older adults and may require adjustment.

The nine one-factor CFA models were fitted to the data for each proposed scale. Based on the criteria for factor loadings greater than 0.4 and less than 0.95 , each item clearly loaded on its own factor, with only one of the 44 items loading $<0.4$. ("7.5 Find out what health care services you are entitled to," 0.20) In the meantime, the difficulty level of this item was also the largest in this questionnaire. We can guess that this item is not applicable to older adults regardless of content or difficulty level. There is also an effect on the reliability of scale 7 .

The majority of the Chinese HLQ items loaded highly on their respective factors and the scales have good reliability. The fit of single-factor to the data was generally good, indicating scale homogeneity. Cronbach's $\alpha>0.70$ is a high confidence standard [41]. In the single-factor model, all Cronbach's $\alpha$ in this study were $>0.75$. Regarding the composite reliability, according to Bagozzi and Yi (1989) [42], composite reliability should be greater than 0.6 . Most of the composite reliability value in this study was greater than 0.80 ; the minimum value was 0.74 . Therefore, the internal reliability of nine single-factor models was considered good. These findings are in the same range as those observed in the original psychometric studies of the English HLQ.

The Spearman correlation coefficient shown in Table 3 indicates that the HLQ has good criterion validity. Although it was lowly correlated, the reason may be that there is a lack of gold standard health literacy scale in China, so the quality of life scale affected by health literacy was selected. Despite this, SF-12 is still a recognized measurement tool and is used as a standard for analyzing the effectiveness of Chinese HLQ. Although presented as low correlation, the validity of HLQ measurements is also demonstrated to a certain extent. Scales 1-6 showed clear discriminant validity. However, the correlations of scales $2,5,6,7,8$, and 9 were $>0.80$, showing that the discriminant validity of these scales was not determined clearly. The high correlation of scales 7, 8, 9 has been shown in previous studies in the United Kingdom, Denmark, and Germany. A Victorian HLQ-related community application study showed that scales 6-9 has a strong correlation, which is consistent in this study [11, 28, 38, 43].

A nine-factor Bayesian model with small variance priors for both cross-loadings and residual correlations comparably fitted the data well, representing the hypothesized factor structure. This model was also used to investigate the discriminant validity of the scales. As for the statistically significant non-target factor loadings, there are three loadings $>0.29$. The strongest of non-target loading is the item P1Q21 (i.e., "There are things that I do regularly to make myself healthier") under scale " 8 . Ability to find good health information. The collection of information is essential before any action. It is easy to understand the 
Table 5 Factor Loadings - Nine-factor Model of the HLQ

\begin{tabular}{|c|c|c|c|c|c|c|c|c|c|}
\hline Item & 1.Understood & $\begin{array}{l}\text { 2..Sufficient } \\
\text { information }\end{array}$ & $\begin{array}{l}\text { 3.Active } \\
\text { management }\end{array}$ & $\begin{array}{l}\text { 4.Social } \\
\text { support }\end{array}$ & 5.Appraisal & $\begin{array}{l}\text { 6.Active } \\
\text { engagement }\end{array}$ & 7.Navigate & $\begin{array}{l}\text { 8.Good } \\
\text { information }\end{array}$ & $\begin{array}{l}\text { 9.Understand } \\
\text { information }\end{array}$ \\
\hline P1Q2 & 0.80 & 0.18 & & & & & & -0.29 & \\
\hline P1Q8 & 0.80 & & & & & & & & \\
\hline P1Q17 & 0.78 & & 0.20 & & & & & & \\
\hline P1Q22 & 0.75 & & & & & & & -0.14 & \\
\hline P1Q1 & & 0.74 & & & & & & & \\
\hline P1Q10 & & 0.53 & 0.15 & & & & & & \\
\hline P1Q14 & 0.14 & 0.58 & & & & & 0.26 & & \\
\hline P1Q23 & & 0.60 & & & & & & & \\
\hline P1Q6 & & 0.16 & 0.76 & & & & & & 0.32 \\
\hline P1Q9 & & & 0.57 & & & & & & \\
\hline P1Q13 & & 0.14 & 0.63 & & & & & & \\
\hline P1Q18 & & & 0.68 & & & & & & \\
\hline P1Q21 & & & 0.63 & & & & & 0.34 & \\
\hline P1Q3 & & & & 0.77 & -0.14 & -0.22 & & & \\
\hline P1Q5 & & & & 0.69 & & & -0.19 & & \\
\hline P1Q11 & & 0.13 & & 0.81 & & & & & \\
\hline P1Q15 & & & & 0.82 & & -0.28 & & & \\
\hline P1Q19 & & & & 0.86 & & -0.24 & & & \\
\hline P1Q4 & & & & & 0.75 & & & & \\
\hline P1Q7 & & & & & 0.69 & & & & \\
\hline P1Q12 & & & & & 0.62 & & & & \\
\hline P1Q16 & & & & & 0.57 & & & & \\
\hline P1Q20 & 0.18 & & & 0.16 & 0.20 & & -0.29 & & \\
\hline P2Q2 & & & & & & 0.73 & & & \\
\hline P2Q4 & & & & & & 0.57 & & & \\
\hline P2Q7 & & & & & & 0.62 & & & \\
\hline P2Q15 & & & & & & 0.69 & & & \\
\hline P2Q20 & & & & & & 0.46 & 0.13 & & \\
\hline P2Q1 & & & & 0.26 & & & 0.64 & & \\
\hline P2Q8 & 0.15 & & 0.25 & & & & 0.42 & & \\
\hline P2Q11 & & & & & & & 0.66 & & \\
\hline P2Q13 & -0.19 & & & 0.25 & & 0.14 & 0.40 & 0.15 & \\
\hline P2Q16 & & & & & & & 0.37 & & \\
\hline P2Q19 & & & -0.27 & & & & 0.42 & & \\
\hline P2Q3 & & & & 0.22 & & & & 0.75 & \\
\hline P2Q6 & & 0.31 & & 0.14 & -0.24 & & & 0.71 & \\
\hline P2Q10 & & & & & & & & 0.47 & \\
\hline P2Q14 & & & 0.18 & & & & & 0.58 & \\
\hline P2Q18 & & & -0.15 & & & & & 0.47 & \\
\hline P2Q5 & & & & & & & & & 0.72 \\
\hline P2Q9 & & & 0.19 & & & & & & 0.48 \\
\hline P2Q12 & & & & & & & & & 0.56 \\
\hline P2Q17 & & & & -0.20 & & & & & 0.62 \\
\hline P2Q21 & & & & & & & & & 0.58 \\
\hline
\end{tabular}


association between them. The most complex factor structure was P2Q13, which is different from Victoria's. The item P1Q16, which was the most complex factor structure in Victoria, was only significantly related to its hypothetical target structure in our results [37], which is presumably owing to cultural differences. However, it is important to note that all target loadings on these and all other scales were higher than any statistically significant non-target loadings.

From this point of view, both the original and other translated versions of the HLQ scale have powerful psychometric attributes. This robustness is related to the sensitivity of group differences related to illness of the original scale and depends on the multi-dimensional selection of the high-quality items [11]. Therefore, it can be inferred that the Chinese HLQ is a good evaluation tool of the health literacy of older adults, resulting in more in-depth and multidimensional.

\section{Limitations}

First, compared with other studies employing CFA, the sample size in this study was relatively small because of factors related to education and age; older adults had difficulty and took a long time filling out the questionnaire. Moreover, previous studies have shown that reading skills are worse among the older population. Nevertheless, the sample size in this study is considered sufficient based on the formula for calculating sample size by scale items [43]. Second, the results of this study showed that the difficulty level of some items is high for older adults. Future research can adjust and verify the content items. Finally, although this study aimed to provide evidence to support the valid use of the HLQ among Chinese older adults, it was limited by the data provided by the participants recruited from the six districts in Changsha City, China. Future research can carry out further investigations and empirical studies on a large scale in mainland China.

\section{Conclusion}

In China, health literacy is expected in the health system. However, there is no scale that is designed to determine the characteristics of older adults. This study showed that the Chinese version of the HLQ has strong construct and content validity and high composite reliability when applied to older adults. The nine-scale HLQ is now available to Chinese older adults, providing a more powerful multidimensional approach to assessing health literacy. The results of this study contribute to health literacy research by providing a basis for the investigation and policy formulation of health literacy for and evidence of health literacy of older adults.

\section{Abbreviations}

BSEM: Bayesian structural equation modeling; CFA: Confirmatory factor analysis; HL: Health Literacy Questionnaire; PPP: Posterior-Predictive-P value

\section{Acknowledgments}

The authors wish to thank Zeng Hui, Yu Renhe, and all the experts and scholars who had provided help. We also wish to thank all the staff involved in the study and the seniors who cooperated with the survey.

\section{Authors' contributions}

The overall study design was led by YWH and TTR. TTR led the cultural debugging. YWH led the CFA. TTW and ZHG contributed to data collection. YWH conducted the statistical analyses reported here and led the interpretation of results. YWH wrote the initial draft, QYY and TTR contributed to redrafting and approving the final draft. All authors read and approved the final manuscript.

\section{Funding}

This study was funded with Hunan Provincial Science and Technology Department Science and Technology Innovation Platform and Talent Plan (2017TP1004). The funding provided assistance in the questionnaire collection process and the language editing service of the manuscript.

\section{Availability of data and materials}

The data and material in this study are available from the corresponding author on reasonable request.

Ethics approval and consent to participate

Ethics approval was obtained from the Ethics Committee of the Xiangya Nursing School, Central South University (Grant No.2017028). Written informed consent was obtained from all patients taking part in face-to-face interviews.

\section{Consent for publication}

Not applicable.

\section{Competing interests}

The authors declare that they have no competing interests.

Received: 27 November 2018 Accepted: 28 August 2019

Published online: 04 September 2019

\section{References}

1. World Health Organization. Health promotion glossary. Health Promot Int. 1998;13(4):349-64

2. Batterham RW, Hawkins M, Collins PA, Buchbinder R, Osborne RH. Health literacy: applying current concepts to improve health services and reduce health inequalities. Public Health. 2016;132:3-12.

3. Ying W, Lu W, Cai Z, Bao L, Pu A, Ai Z. Prevalence and risk factors of low health literacy: a community-based study in shanghai, China. Int J Environ Res Public Health. 2017;14(6):628.

4. Heide IVD, Poureslami I, Mitic W, Shum J, Rootman I, Fitzgerald JM. Health literacy in chronic disease management: a matter of interaction. J Clin Epidemiol. 2018;102:134-8.

5. Schrauben SJ, Wiebe DJ. Health literacy assessment in developing countries: a case study in Zambia. Health Promot Int. 2017;32(3):dav108.

6. Kickbusch I, Pelikan JM, Apfel F, Tsouros AD. Health literacy: the solid facts. Copenhagen: WHO Regional Office for Europe; 2013.

7. Norgaard O, Sorensen K, Maindal HT, Kayser L. Measuring health literacy can improve communication in health care. Ugeskr Laeger. 2014;176:37-9.

8. Liu H, Zeng H, Shen Y, Zhang F, Sharma M, Lai W, et al. Assessment tools for health literacy among the general population: a systematic review. Int J Environ Res Public Health. 2018;15(8):1711

9. Zamora $\mathrm{H}$, Clingerman EM. Health literacy among older adults: a systematic literature review. J Gerontol Nurs. 2011;37(10):41-51.

10. Berkman ND, Sheridan SL, Donahue KE, Halpern DJ, Crotty K. Low health literacy and health outcomes: an updated systematic review. Ann Intern Med. 2011;155(2):97.

11. Maindal HT, Kayser L, Norgaard O, Bo A, Elsworth GR, Osborne RH. Cultural adaptation and validation of the health literacy questionnaire (hlq): robust nine-dimension Danish language confirmatory factor model. Springerplus. 2016;5(1):1232.

12. Baker DW, Gazmararian JA, Sudano J, Patterson M. The association between age and health literacy among elderly persons. J Gerontol. 2000;55(6):S368. 
13. Mark K, Elizabeth G, Ying J, Christine P. The health literacy of America's adults: results from the 2003 national assessment of adult literacy. Nces 2006-483. Natl Center Educ Stat. 2006:39(10):685-7.

14. Ping W, An MQ, Xuan TM, Yang TX, Hua LY, Ling Q, et al. Survey on the status of health literacy of Chinese residents in 2008. Chin J Health Educ. 2010;26(04): 243-6.

15. Kripalani S, Jacobson TA, Mugalla IC, Cawthon CR, Niesner KJ, Vaccarino V. Health literacy and the quality of physician-patient communication during hospitalization †. J Hosp Med An Off Publ Soc Hospital Med. 2010;5(5):269.

16. Ishikawa H, Kiuchi T. Health literacy and health communication. Biopsychosoc Med. 2010;4(1):18.

17. Heijmans M, Waverijn G, Rademakers J, Van d VR, Rijken M. Functional, communicative and critical health literacy of chronic disease patients and their importance for self-management. Patient Educ Couns. 2015;98(1):41-8.

18. Jordan JE, Osborne RH, Buchbinder R. Critical appraisal of health literacy indices revealed variable underlying constructs, narrow content and psychometric weaknesses. J Clin Epidemiol. 2011;64(4):366-79.

19. Buchbinder R, Batterham R, Ciciriello S, Newman S, Horgan B, Ueffing E, et al. Health literacy: what is it and why is it important to measure. J Rheumatol. 2011;38(8):1791-7.

20. Jordan JE, Buchbinder R, Osborne RH. Conceptualising health literacy from the patient perspective. Patient Educ Couns. 2010;79(1):36-42.

21. OuYang $Y$, Wang $X$, Yang $C$, et al. Research progress on health literacy assessment tools for the elderly. Nurs J Chin People's Liber Army. 2018; 35(2):39-43.

22. Batterham RW, Buchbinder R, Beauchamp A, Dodson S, Elsworth GR, Osborne RH. The optimising health literacy (Ophelia) process: study protocol for using health literacy profiling and community engagement to create and implement health reform. BMC Public Health. 2014;14(1):1-10.

23. Goeman D, Conway S, Norman R, Morley J, Weerasuriya R, Osborne RH, et al. Optimising health literacy and access of service provision to community dwelling older people with diabetes receiving home nursing support. J Diabetes Res. 2016;2016(1):694.

24. Buchbinder R, Batterham R, Elsworth G, Dionne CE, Irvin E, Osborne RH. A validity-driven approach to the understanding of the personal and societal burden of low back pain: development of a conceptual and measurement model. Arthritis Res Ther. 2011;13(5):R152.

25. Hawkins M, Gill SD, Batterham R, Elsworth GR, Osborne RH. The health literacy questionnaire (hlq) at the patient-clinician interface: a qualitative study of what patients and clinicians mean by their hlq scores. BMC Health Serv Res. 2017;17(1):309.

26. Raykov T. Coefficient alpha and composite reliability with interrelated nonhomogeneous items. Appl Psychol Measur. 1998;22(4):375-85.

27. Raykov T. Reliability if deleted, not 'alpha if deleted': evaluation of scale reliability following component deletion. Brit J Math Stat Psy. 2007;60:201-16.

28. Browne $M$, Cudeck R. Alternative ways of assessing model fit. In: Bollen K, Long J, editors. Testing structural equation models. Newbury Park, London, New Delhi: Sage Publications; 1993.

29. Gonzálezromá $V$. The measurement of engagement and burnout: a two sample confirmtory factor analytic approach; 2002.

30. Ayearst LE, Harsanyi Z, Michalko KJ. The pain and sleep questionnaire threeitem index (psq-3): a reliable and valid measure of the impact of pain on sleep in chronic nonmalignant pain of various etiologies. Pain Res Manage J Can Pain Soc. 2012;17(4):281.

31. McDonald RP, Ho MR. Principles and practice in reporting structural equation analyses. Psychol Methods. 2002;7:64-82.

32. Hu L, Bentler PM. Cutoff criteria for fit indexes in covariance structure analysis: conventional criteria versus new alternatives. Struct Equ Modeling. 1999;6:1-55.46.

33. Marsh HW, Muthén B, Asparouhov T, Lüdtke $O$, Robitzsch A, Morin AJS, et al. Exploratory structural equation modeling, integrating cfa and efa: application to students $\backslash$ evaluations of university teaching. Struct Equ Model Multidiscip J. 2009;16(3):439-76.

34. Marsh HW, Lüdtke O, Muthén B, Asparouhov T, Morin AJ, Trautwein U, et al. A new look at the big five factor structure through exploratory structural equation modeling. Psychol Assess. 2010;22(3):471-91.

35. Muthén B, Asparouhov T. Bayesian structural equation modeling: a more flexible representation of substantive theory. Psychol Methods. 2012;17:313-35.

36. Asparouhov T, Muthén B. Multiple-group factor analysis alignment. Struct Equ Model Multidiscip J. 2014;21(4):495-508
37. Elsworth GR, Beauchamp A, Osborne RH. Measuring health literacy in community agencies: a bayesian study of the factor structure and measurement invariance of the health literacy questionnaire (hlq). BMC Health Serv Res. 2016;16(1):508.

38. Hou S. Has the internet replaced traditional media and human as a major health information source among Chinese middle- and older-age adults; 2013. Apha

39. Levin-Zamir D, Bertschi I. Media health literacy, eHealth literacy, and the role of the social environment in context. Int J Environ Res Public Health. 2018;15(8): 1643

40. Bo A, Friis $\mathrm{K}$, Osborne RH, Maindal $\mathrm{H}$. National indicators of health literacy: ability to understand health information and to engage actively with healthcare providers - a population-based survey among Danish adults. BMC Public Health. 2014;14:1095. https://doi.org/10.1186/1471-2458-14-1095.

41. Guilford JP. Psychometric Methods (2nd Edn). New York: McGraw-Hill; 1954.

42. Yi Y, Singh S, Marketing DO, NAD A, NAD A, Arbor A. On the use of structural equation models in experimental designs: two extensions. J Mark Res. 1989;26(3):271-84

43. Osborne RH, Batterham RW, Elsworth GR, Hawkins M, Buchbinder R. The grounded psychometric development and initial validation of the health literacy questionnaire (HLQ). BMC Public Health. 2013;16(7):658.

\section{Publisher's Note}

Springer Nature remains neutral with regard to jurisdictional claims in published maps and institutional affiliations.
Ready to submit your research? Choose BMC and benefit from:

- fast, convenient online submission

- thorough peer review by experienced researchers in your field

- rapid publication on acceptance

- support for research data, including large and complex data types

- gold Open Access which fosters wider collaboration and increased citations

- maximum visibility for your research: over $100 \mathrm{M}$ website views per year

At $\mathrm{BMC}$, research is always in progress.

Learn more biomedcentral.com/submissions 